\title{
Chinese highly pathogenic porcine reproductive and respiratory syndrome virus exhibits more extensive tissue tropism for pigs
}

\author{
Limin Li, Qian Zhao, Xinna Ge, Kedao Teng, Yu Kuang, Yanhong Chen, Xin Guo* and Hanchun Yang*
}

\begin{abstract}
Background: The highly pathogenic porcine reproductive and respiratory syndrome virus (PRRSV) emerging in China exhibits high fatality to pigs. However, the mechanism related to the increased pathogenicity of the virus remains unclear. In the present study, the differences in tissue tropism between the highly pathogenic PRRSV strain (JXwn06) and the low pathogenic PRRSV strain (HB-1/3.9) were investigated using PRRSV-specific immunohistochemistry (IHC) staining to provide evidence for elucidating possible mechanism of the pathogenicity of Chinese highly pathogenic PRRSV.

Findings: IHC examination showed that PRRSV antigen in the tissues including spleen, tonsil, thymus, kidney, cerebellum, stomach, small intestine, large intestine, turbinal bone and laryngeal cartilage was positive in more pigs inoculated with JXwn06 than HB-1/3.9, and the tissues including trachea, esophagus, liver, mandibular gland and thyroid gland were positive for viral antigen in the pigs inoculated with JXWn06, but not in the pigs inoculated with HB-1/3.9. Meanwhile, we observed that epithelium in tissues including interlobular bile duct in liver, distal renal tubule of kidney, esophageal gland and tracheal gland were positive for viral antigen only in JXwn06-inoculated pigs, and epithelium of gastric mucosa and fundic gland, and intestinal gland were positive for viral antigen in both JXwn06- and HB-1/3.9-inoculated pigs, using monoclonal antibodies to N and Nsp2 proteins.
\end{abstract}

Conclusions: Taken together, these findings indicate that the highly pathogenic PRRSV JXwn06 displays an expanded tissue tropism in vivo, suggesting this may contribute to its high pathogenicity to pigs.

Keywords: Porcine reproductive and respiratory syndrome virus, High pathogenicity, Tissue tropism, Immunohistochemistry

\section{Findings}

Porcine reproductive and respiratory syndrome (PRRS) has become a well-recognized global swine disease [1,2] since it was first reported as a "mystery swine disease" in the United States in 1987 [3]. The disease is predominantly characterized by reproductive failure in sows, preweaning mortality, and respiratory disorders in pigs of all ages $[4,5]$, resulting in great economic losses for the swine industry [6].

Porcine reproductive and respiratory syndrome virus (PRRSV), the causative agent of the disease, is a member

\footnotetext{
*Correspondence: guoxin@cau.edu.cn; yanghanchun1@cau.edu. cn Key Laboratory of Animal Epidemiology and Zoonosis of Ministry of Agriculture, College of Veterinary Medicine and State Key Laboratory of Agrobiotechnology, China Agricultural University, No. 2 Yuanmingyuan West Road, Haidian District, Beijing 100193, China
}

of the family Arteriviridae [7]. Previous studies have indicated that PRRSV infects macrophages in some tissues of pigs and mainly replicates within macrophages in the respiratory (lung) and lymphoid systems $[8,9]$. Swine pulmonary alveolar macrophages are the predominantly cells for virus replication [10,11] although immature sperm cells and epithelial pulmonary cells were recognized to also be infected by PRRSV [12]. A number of investigations revealed that the largest amount of viral antigen and/or nucleic acid could be observed in lungs and lymph nodes of the pigs following PRRSV infection [10,12-15]. Therefore, PRRSV mainly has a tropism for the respiratory and lymphoid tissues of pigs. In addition, it has been shown that the tissue tropism, the cell types in which PRRSV antigen was detected and distribution of PRRSV antigen-positive cells within particular tissues

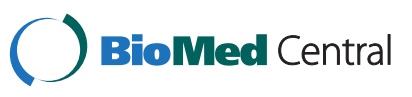

(c) 2012 Li et al.; licensee BioMed Central Ltd. This is an Open Access article distributed under the terms of the Creative Commons Attribution License (http://creativecommons.org/licenses/by/2.0), which permits unrestricted use, distribution, and reproduction in any medium, provided the original work is properly cited. 
and organs are generally similar among PRRSV strains with differences in virulence, even though the isolates with increased virulence often replicate better in vivo than the less virulent isolates $[14,16]$.

Since 2006, the clinical outbreaks of atypical PRRS caused by a highly pathogenic PRRSV with unique 30-amino-acid (30-aa) deletion in its nonstructural protein 2 (Nsp2)-coding region were widespread in major pig-producing areas of China [17]. These outbreaks have brought great economic losses to the swine industry in China due to the high mortality and morbidity in the affected pig herds. To date, all the evidences have pointed to the conclusion that the highly pathogenic PRRSV with the 30-aa deletion in Nsp2-coding region is the causative agent of atypical PRRS in China, and the virus has marked characteristic of high pathogenicity aside from the genomic characteristic of 30 -aa deletion in Nsp2, compared with previous Chinese strains [18-20]. Although our recent findings have revealed that the 30-aa deletion in Nsp2 is not related to its virulence [20], the mechanisms associated with high pathogenicity of the virus for pigs remain unclear.

In the present study, we observed the distribution of viral antigen in tissues of the pigs experimentally infected with a highly pathogenic PRRSV strain (JXwn06) and simultaneously analyzed the tissue tropism of the virus in comparison with a low pathogenic strain (HB-1/3.9), in order to provide valuable information that might be helpful for explaining differences in the virulence of these viruses.

Two PRRSV strains that differ in virulence, JXwn06 and HB-1/3.9, were used for the study. Starin JXwn06, a highly pathogenic strain that cause fatality for pigs, was isolated from an intensive pig farm with an atypical PRRS outbreak in the Jiangxi province of China in 2006 [20]. HB-1/3.9, a low-virulence and adapted strain in MARC-145 cells that induce no fatality for pigs, was derived from HB-1(sh)/2002 isolated in 2002 [21]. The genomic sequence of JXwn06 shared $97.4 \%$ nucleotide identity with HB-1/3.9. The marked genomic difference between the two viruses is that a 30-aa deletion exists in Nsp2-coding region of JXwn06, not in HB-1/3.9 [20]. Thirteen 6-week-old specific-pathogen free (SPF) Landrace piglets, free of PRRSV, porcine circovirus type 2, classical swine fever virus, porcine parvovirus, pseudorabies virus, swine influenza virus and Mycoplasma hyopneumoniae, were obtained from the Beijing Center for SPF Swine Breeding and Management. The animals were allocated randomly to three groups-JXwn06inoculated group $(n=5)$, HB-1/3.9-inoculated group $(n=5)$ and control group $(n=3)$. Each group was housed separately in a different isolation room, with individual ventilation. Each piglet in the JXwn06-inoculated group or HB-1/3.9-inoculated group was administered intranasally at a dose of $2 \times 10^{5.0}$ TCID $_{50}$ of the fifthpassage viral cultures on MARC-145 cells. The piglets in the control group were exposed in the same manner to uninfected MARC-145 cell culture supernatant. Following the inoculations, all animals were clinically observed and rectal temperatures were recorded daily until death or necropsy. Serum samples were collected prior to inoculation and from day 1 onwards post-inoculation (PI) for virus detection by RT-PCR using the primers (the forward primer: 5'-CAAATAACAACGGCAAGCAG-3'; the reverse primer: 5'-AAACTCCACAGTGTAACT TAT-3') to amplify ORF7 gene of PRRSV. Tissues of JXwn06-inoculated pigs were collected when the pigs died. HB-1/3.9-inoculated pigs were euthanized for necropsy on day 7 PI when the peak of viremia occurred during a three-week-experimental period in our previous study [20], and their tissues were collected. Also, on day $7 \mathrm{PI}$, the control pigs were euthanized for necropsy and their tissues were collected. Total collected tissues are listed in Table 1. All the tissues were fixed in $4 \%$ neutral

\section{Table 1 Distribution and staining intensity of PRRSV antigen-positive tissues by IHC using monoclonal antibody to $\mathbf{N}$ protein}

\begin{tabular}{|c|c|c|}
\hline \multirow[t]{2}{*}{ Tissues } & \multirow{2}{*}{$\begin{array}{c}\text { JXwn06-inoculated } \\
\text { pigs }\end{array}$} & \multirow{2}{*}{ 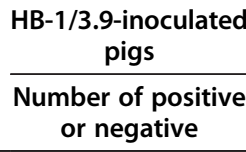 } \\
\hline & & \\
\hline Lung & $+5 / 5$ & $+5 / 5$ \\
\hline Trachea & $+5 / 5$ & $-5 / 5$ \\
\hline Turbinal bone & $+5 / 5$ & $+3 / 5$ \\
\hline Spleen & $+5 / 5$ & $+3 / 5$ \\
\hline Tonsil & $++5 / 5$ & $+4 / 5$ \\
\hline Thymus & $+3 / 5$ & $+1 / 5$ \\
\hline Mesenteric lymph node & $+5 / 5$ & $+5 / 5$ \\
\hline Inguinal lymph node & $+5 / 5$ & $+5 / 5$ \\
\hline Laryngeal cartilage & $+5 / 5$ & $+2 / 5$ \\
\hline Esophagus & $++5 / 5$ & $-5 / 5$ \\
\hline Stomach & $++5 / 5$ & $+1 / 5$ \\
\hline Small intestine & $++5 / 5$ & $++1 / 5$ \\
\hline Large intestine & $++5 / 5$ & $++1 / 5$ \\
\hline Liver & $+5 / 5$ & $-5 / 5$ \\
\hline Cerebrum & $+4 / 5$ & $-5 / 5$ \\
\hline Cerebellum & $+3 / 5$ & $+1 / 5$ \\
\hline Kidney & $++5 / 5$ & $+1 / 5$ \\
\hline Mandibular gland & $++5 / 5$ & $-5 / 5$ \\
\hline Thyroid gland & $++5 / 5$ & $-5 / 5$ \\
\hline Heart & $-5 / 5$ & $-5 / 5$ \\
\hline
\end{tabular}

Notes: All the tissue sections were observed under 400x in magnification, and the average positive cells in five visual fields randomly of each section were counted. + represents $<10$ positive cells; ++ means $>10$ positive cells; - denotes no positive cell. 
buffered polyoxymethylene for immunohistochemistry (IHC) examination of viral antigen. The experimental researches on animals in this study have followed international recognized guidelines, and have been approved by The Beijing Municipal Committee of Laboratory Animal Management and The Ethics Committee of China Agricultural University.

From day 2 PI onwards, the body temperatures of pigs in JXwn06-inoculated group started to rise and had a mean peak of $41.3^{\circ} \mathrm{C}$ on day 4 PI, while the pigs in HB1/3.9-inoculated group showed slightly an elevated body temperature from day 3 PI onwards (Figure 1A). The body temperatures of pigs inoculated with JXwn06 were significantly higher than those of pigs inoculated with HB-1/3.9 from on day 3 PI onwards $(p<0.01)$. The pigs inoculated with JXwn06 showed marked clinical signs, including depression, anorexia and lethargy, rubefaction on skin and in ears, respiratory distress, shivering and diarrhea, and died within 5-10 days PI (Figure 1B), whereas the pigs inoculated with $\mathrm{HB}-1 / 3.9$ presented no obvious clinical manifestations within 7 days PI. All the serum samples from both JXwn06- and HB-1/3.9inoculated pigs on days 3 to 7 PI were positive for viral nucleic acid detection by RT-PCR (Table 2). It is worthy to note that the virus could be detected earlier on day $1 \mathrm{PI}$, in the group inoculated with the highly pathogenic JXwn06. Our previous data also showed the virus titers in the serum of the pigs infected with JXwn06 were much higher than those of the pigs infected with HB-1/3.9 [20]. For the control pigs, no body temperature elevation or clinical signs were observed, and no virus was detected in serum samples.

The monoclonal antibody against PRRSV $\mathrm{N}$ protein (diluted 1:100) [22] was used for the detection of PRRSV antigen in the tissues of the inoculated pigs. The number and intensity of PRRSV antigen-positive tissues between JXwn06-inoculated pigs and HB-1/3.9-inoculated pigs are summarized in Table 1 . The results showed that PRRSV antigen was detected in trachea, esophagus, liver,
Table 2 Viremia detection of the inoculated pigs by RT-PCR

\begin{tabular}{lll}
\hline DPI & JXwn06-inoculated pigs & HB-1/3.9-inoculated pigs \\
\hline 0 & $-5 / 5^{\text {a }}$ & $-5 / 5$ \\
1 & $+5 / 5$ & $-5 / 5$ \\
2 & $+5 / 5$ & $-5 / 5$ \\
3 & $+5 / 5$ & $+4 / 5$ \\
4 & $+5 / 5$ & $+5 / 5$ \\
5 & $+4 / 4$ & $+5 / 5$ \\
6 & $+4 / 4$ & $+5 / 5$ \\
7 & $+3 / 3$ & $+5 / 5$ \\
8 & $+3 / 3$ & NA \\
9 & $+1 / 1$ & NA \\
10 & NA & NA
\end{tabular}

a positive or negative number / animal number, + positive, - negative. b not applicable.

cerebrum, mandibular gland and thyroid gland in JXwn06-inoculated pigs; while, no positive signals were found in these tissues in HB-1/3.9-inoculated pigs. The positive tissues including liver, mandibular gland, cerebrum, and esophagus of JXwn06-inoculated pigs are shown in Figure 2. Lung, turbinal bone, laryngeal cartilage, stomach, intestine, cerebellum, kidney, spleen, tonsil, thymus and lymph node were the tissues in which positive signals were detected both in JXwn06inoculated pigs and HB-1/3.9-inoculated pigs. It is noteworthy that the positive rate of PRRSV antigen in JXwn06-inoculated pigs was higher than in HB-1/3.9inoculated pigs for turbinal bone (5/5 vs. $3 / 5)$, laryngeal cartilage ( $5 / 5$ vs. $2 / 5)$, small intestine ( $5 / 5$ vs. $1 / 5)$, large intestine $(5 / 5$ vs. $1 / 5)$, cerebellum $(3 / 5$ vs. $1 / 5)$, spleen (5/5 vs. $3 / 5)$ and thymus (3/5 vs. $1 / 5)$; and more positive cells were observed in stomach, kidney and tonsil of JXwn06-inoculated pigs compared to HB-1/3.9-inoculated pigs.
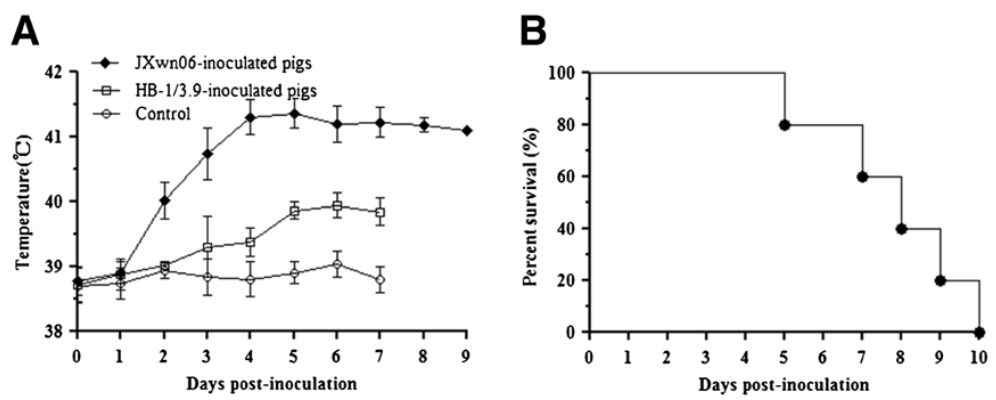

Figure 1 Rectal temperature and the survival curve of the inoculated pigs. Shown are rectal temperature change $(\mathbf{A})$ and survival rates $(\mathbf{B})$ of the JXwn06-inoculated pigs. The data for body temperature are means \pm standard deviations (error bars), except for the temperature of the one JXwn06-inoculated pig that survived on day 9 PI. 


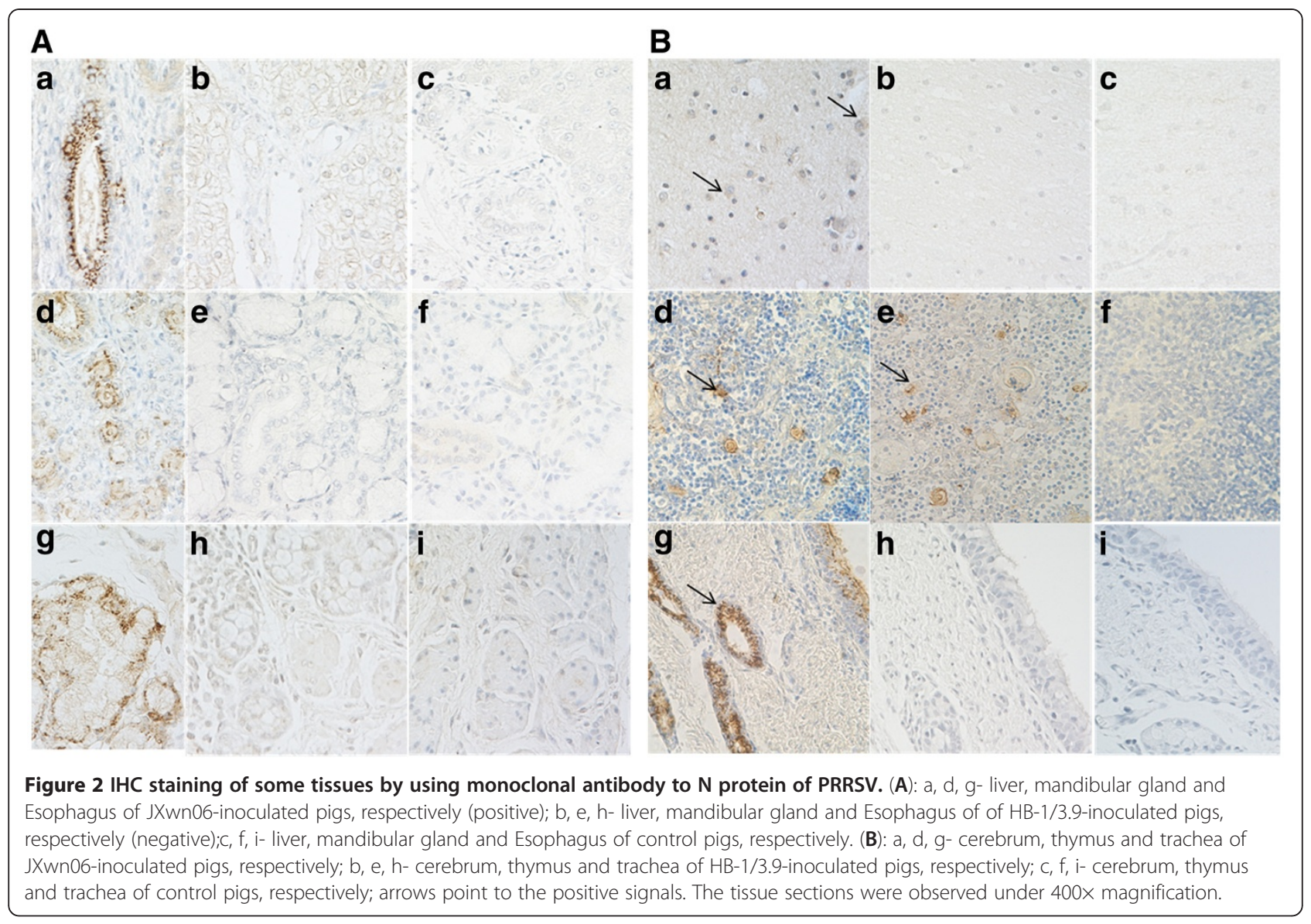

PRRSV antigen-positive cells observation showed that both the highly and low pathogenic virus has a tropism for macrophages in the respiratory and lymphoid systems of the inoculated pigs. Lymph node, tonsil, spleen and thymus were the tissues with positive signals mainly in macrophages which were identified both in JXwn06- and HB-1/3.9-inoculated pigs, and the positive cells were mostly distributed in medullar substance and paracortical area in lymph organs (Additional file 1). In addition, strong positive signals could be observed in epithelium of some of tissues of both JXwn06- and HB-1/3.9-inoculated pigs. Besides pulmonary alveolar macrophages, bronchial epithelium was positive in lung of the inoculated pigs. In stomach, small intestine, large intestine, laryngeal cartilage and turbinal bone, viral antigen was mainly seen in the mucous membrane epithelium or glandular epithelium. The epithelium of interlobular bile duct in liver, distal renal tubule in kidney, esophageal gland and tracheal gland were found positive only in JXwn06-inoculated pigs. These findings indicate that the highly pathogenic PRRSV JXwn06 exhibits an expanded tissue tropism in vivo compared to the low pathogenic PRRSV HB-1/3.9, suggesting that JXwn06 has an increased ability to replicate in vivo compared to HB-1/3.9. In addition, the hearts were negative for viral antigen in both JXwn06- and HB-1/3.9inoculated pigs, this contradicts with earlier reports that macrophages and endothelial cells in heart could be infected by PRRSV $[8,10]$, suggesting this might be due to the pathogenicity differences among the virus strains. No positive signals were observed in any tissues from the control pigs or when PBS or normal mouse sera were used as a substitution for the primary antibody for IHC staining.

PRRSV antigen-positive tissues detected with monoclonal antibody against $\mathrm{N}$ protein were further stained using monoclonal antibody against Nsp2 (diluted 1:400) [23]. Positive signals detected using the two antibodies were consistent. The results showed that the positive signals were observed not only in macrophages mainly in lymphoid organs, but also in epithelium including esophageal gland, gastric mucous membrane and fundic gland, intestinal gland, interlobular bile duct in liver and mandibular gland as well as renal tubule in kidney. It could be speculated that this may be due to the accumulation of viral particles within the epithelium of these tissues or the consequence resulting from the replication of PRRSV in epithelial cells within these tissues. However, the latter needs to be further confirmed in vitro. 


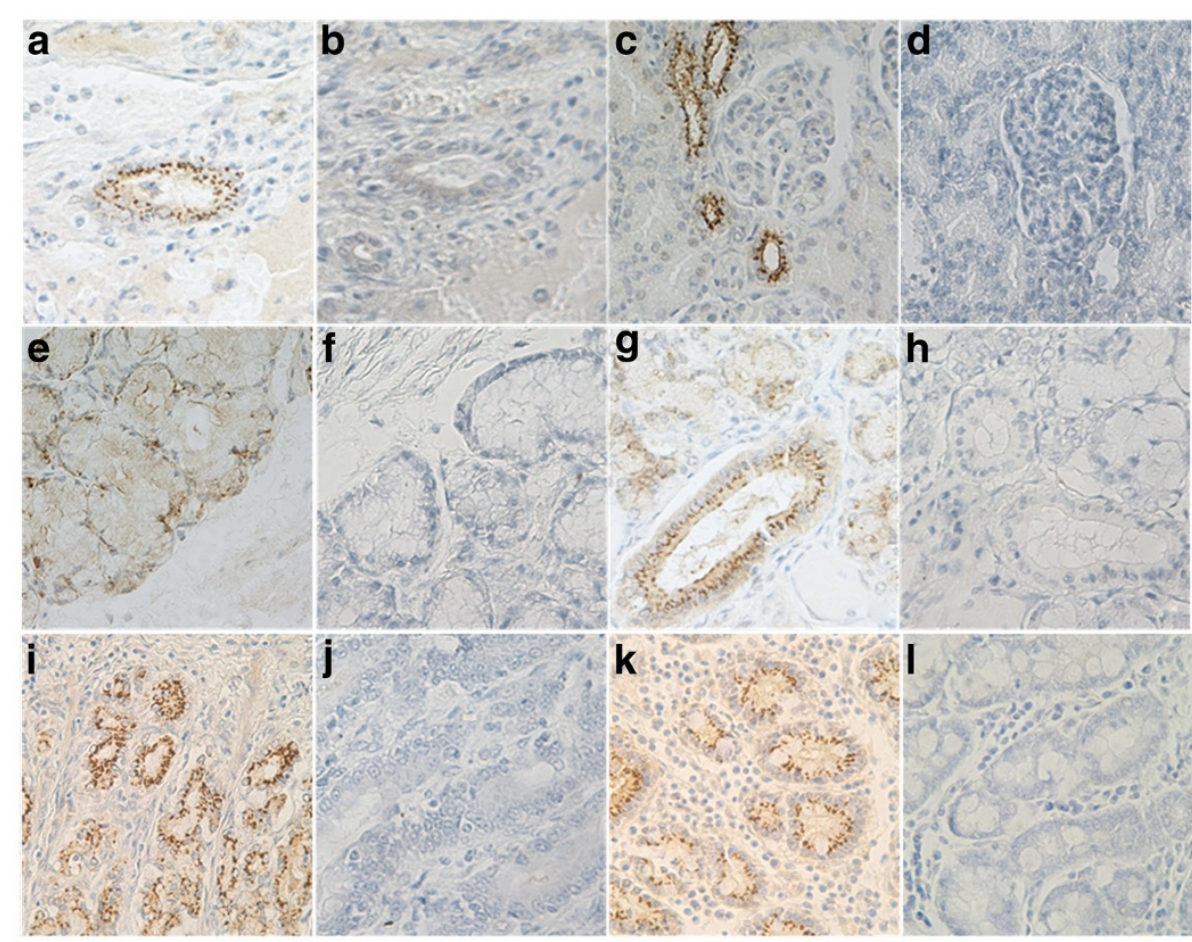

Figure 3 IHC staining of epithelium in tissues by using monoclonal antibody to Nsp2 of PRRSV. a, c, e, g, i, k - the epithelium of interlobular bile duct in liver, distal renal tubule in kidney, esophageal gland and mandibular gland, the epithelium of gastric fundic gland and intestinal gland from JXwn06- inoculated pigs, respectively; $b, d, f, h, j, l$ - the corresponding tissues stained by normal mice sera as primary antibody. The tissue sections were observed under 400x magnification.

Partial tissues with positive signals in epithelium are shown in Figure 3.

Our present findings describe the tissue distribution of viral antigen of a Chinese highly pathogenic strain of PRRSV using IHC. In summary, the highly pathogenic PRRSV emerging in China exhibits an expanded tissue tropism in vivo, suggesting a possible mechanism that contributes to its high pathogenicity for pigs.

\section{Additional file}

Additional file 1: IHC staining of lung and lymph node of the inoculated pigs using monoclonal antibody to $\mathrm{N}$ protein.

\section{Competing interests}

The authors declare that they have no competing interests.

\section{Authors' contributions}

LML carried out animal experiment, performed $I H C$ staining of the tissues and wrote the manuscript. QZ and YHC participated in animal experiment. XNG conducted RT-PCR for PRRSV detection. KDT and YK participated in the preparation of tissue sections and $\mathrm{IHC}$ staining. $\mathrm{XG}$ and $\mathrm{HCY}$ participated in study design and coordination, and revised the manuscript. All authors approved the final manuscript.

\section{Acknowledgements}

This work was supported by the National Natural Science Funds for Distinguished Young Scholars (30825031) from the National Natural Science
Foundation of China, and the earmarked fund for Modern Agro-industry Technology Research System of China (CARS-36).

Received: 18 October 2011 Accepted: 14 September 2012

Published: 17 September 2012

\section{References}

1. Albina E: Epidemiology of porcine reproductive and respiratory syndrome (PRRS): an overview. Vet Microbiol 1997, 55:309-316.

2. Cho JG, Dee SA: Porcine reproductive and respiratory syndrome virus. Theriogenology 2006, 66:655-662.

3. Keffaber K: Reproductive failure of unkwown etiology. Am Assoc Swine Pract Newslett 1989, 1:9.

4. Hopper SA, White ME, Twiddy N: An outbreak of blue-eared pig disease (porcine reproductive and respiratory syndrome) in four pig herds in Great Britain. Vet Rec 1992, 131:140-144.

5. Rossow KD: Porcine reproductive and respiratory syndrome. Vet Pathol 1998, 35:1-20

6. Pejsak Z, Stadejek T, Markowska-Daniel I: Clinical signs and economic losses caused by porcine reproductive and respiratory syndrome virus in a large breeding farm. Vet Microbiol 1997, 55:317-322.

7. Meulenberg JJ, Hulst MM, De-Meijer EJ, Moonen PL, Den-Besten A, DeKluyver EP, Wensvoort G, Moormann RJ: Lelystad virus, the causative agent of porcine epidemic abortion and respiratory syndrome (PEARS), is related to LDV and EAV. Virology 1993, 192:62-72.

8. Halbur PG, Miller LD, Paul PS, Meng XJ, Huffman EL, Andrews JJ: Immunohistochemical identification of porcine reproductive and respiratory syndrome virus (PRRSV) antigen in the heart and lymphoid system of three-week-old colostrum-deprived pigs. Vet Pathol 1995, 32:200-204.

9. Duan $X$, Nauwynck HJ, Pensaert MB: Virus quantification and identification of cellular targets in the lungs and lymphoid tissues of pigs at different time intervals after inoculation with porcine reproductive and respiratory syndrome virus (PRRSV). Vet Microbiol 1997, 56:9-19. 
10. Rossow KD, Benfield DA, Goyal SM, Nelson EA: Chronological immunohistochemical detection and localization of porcine reproductive and respiratory syndrome virus in gnotobiotic pigs. Vet Pathol 1996, 33:551-556.

11. Shibata I, Mori M, Uruno K, Samegai Y, Okada M: In vivo replication of porcine reproductive and respiratory syndrome virus in swine alveolar macrophages and change in the cell population in bronchoalveolar lavage fluid after infection. J Vet Med Sci 1997, 59:539-543.

12. Sur JH, Doster AR, Christian JS, Galeota JA, Will RW, Zimmerman JJ, Osorio FA: Porcine reproductive and respiratory syndrome virus replicates in testicular germ cells, alters spermatogenesis, and induces germ cell death by apoptosis. J Virol 1997, 71:9170-9179.

13. Magar R, Larochelle R, Robinson Y, Dubuc C: Immunohistochemical detection of porcine reproductive and respiratory syndrome virus using colloidal gold. Can J Vet Res 1993, 57:300-304.

14. Halbur PG, Paul PS, Frey ML, Landgraf J, Eernisse K, Meng XJ, Andrews JJ, Lum MA, Rathje JA: Comparison of the antigen distribution of two US porcine reproductive and respiratory syndrome virus isolates with that of the Lelystad virus. Vet Pathol 1996, 33:159-170.

15. Pol JM, Van-Dijk JE, Wensvoort G, Terpstra C: Pathological, ultrastructural, and immunohistochemical changes caused by Lelystad virus in experimentally induced infections of mystery swine disease (synonym: porcine epidemic abortion and respiratory syndrome (PEARS)). Vet $Q$ 1991, 13:137-143.

16. Haynes JS, Halbur PG, Sirinarumitr T, Paul PS, Meng XJ, Huffman EL: Temporal and morphologic characterization of the distribution of porcine reproductive and respiratory syndrome virus (PRRSV) by in situ hybridization in pigs infected with isolates of PRRSV that differ in virulence. Vet Pathol 1997, 34:39-43.

17. Tian $K$, Yu X, Zhao T, Feng Y, Cao Z, Wang C, Hu Y, Chen X, Hu D, Tian X, Liu D, Zhang S, Deng X, Ding Y, Yang L, Zhang Y, Xiao H, Qiao M, Wang B, Hou L, Wang $X$, Yang $X$, Kang $L$, Sun $M$, Jin $P$, Wang $S$, Kitamura $Y$, Yan J, Gao GF: Emergence of fatal PRRSV variants: unparalleled outbreaks of atypical PRRS in China and molecular dissection of the unique hallmark. PLoS One 2007, 2:526.

18. Li Y, Wang X, Bo K, Tang B, Yang B, Jiang W, Jiang P: Emergence of a highly pathogenic porcine reproductive and respiratory syndrome virus in the Mid-Eastern region of China. Vet J 2007, 174(3):577-584.

19. Zhou YJ, Hao XF, Tian ZJ, Tong GZ, Yoo D, An TQ, Zhou T, Li GX, Qiu HJ, Wei TC, Yuan XF: Highly virulent porcine reproductive and respiratory syndrome virus emerged in China. Transbound Emerg Dis 2008, 55:152-164.

20. Zhou L, Zhang J, Zeng J, Yin S, Li Y, Zheng L, Guo X, Ge X, Yang H: The 30amino-acid deletion in the Nsp2 of highly pathogenic porcine reproductive and respiratory syndrome virus emerging in China is not related to its virulence. J Virol 2009, 83:5156-5167.

21. Gao ZQ, Guo X, Yang HC: Genomic characterization of two Chinese iaolates of porcine reproductive and respiratory syndrome virus. Arch Virol 2004, 149:1341-1351.

22. Gu H, Zhang ZZ, Yang HC, Zhao JS: Preparation of the monoclonal antibodies against porcine reproductive and respiratory syndrome virus (PRRSV). Chin J Vet Sci Technol 2000, 30:9-11.

23. Yan Y, Guo X, Ge X, Chen Y, Cha Z, Yang H: Monoclonal antibody and porcine antisera recognized B-cell epitopes of Nsp2 protein of a Chinese strain of porcine reproductive and respiratory syndrome virus. Virus Res 2007, 126:207-215.

doi:10.1186/1743-422X-9-203

Cite this article as: Li et al:: Chinese highly pathogenic porcine reproductive and respiratory syndrome virus exhibits more extensive tissue tropism for pigs. Virology Journal 2012 9:203.

\section{Submit your next manuscript to BioMed Central and take full advantage of:}

- Convenient online submission

- Thorough peer review

- No space constraints or color figure charges

- Immediate publication on acceptance

- Inclusion in PubMed, CAS, Scopus and Google Scholar

- Research which is freely available for redistribution

Submit your manuscript at www.biomedcentral.com/submit
Ciomed Central 